Jpn. J. Genet. (1984) 59, pp. 51-59

\title{
The effect of another population on emigration behavior of a population
}

\author{
By Takashi NARISE and Kenji MIKasa \\ Department of Biology, Josai Dental University \\ Sakado, Saitama 350-02
}

(Received June 29, 1983)

\begin{abstract}
In order to examine the effect of one population on the emigration behavior of another population, seven wild strains of Drosophila melanogaster were used. The SI and KG strain were chosen as tester strain. Single and mixed strain experiments were done, in which two strains were mixed, and three kinds of relative frequencies in the mixtures-0.9, 0.5 , and 0.1 - were utilized. In mixtures of SI and its neighboring populations, emigration activity was decreased in comparison with the strain in the single condition, but the activity increased when distant populations were mixed. The same tendency was observed in mixtures of $\mathrm{KG}$ and other four strains. The increase or decrease in activity was mainly due to the stimulation or diminution of activity in the SI or KG strain. The A/B ratio was also calculated, and it was less than 1.0 in the mixtures of tester strain and its neighbouring populations, while greater than 1.0 in mixtures of tester strain and distant populations. Since A/B ratio is lower when genetically similar populations are mixed, this ratio may be useful to determine genetic relationships among populations.
\end{abstract}

\section{INTRODUCTION}

Under natural conditions, it is considered that animals move freely among populations. Two paramaters of this movement involve distance that members of a population move from their native place and the proportion of that population which move. Dobzhansky and Wright $(1943,1947)$ and Powell et al. (1967) studied the former problem by the release-recapture method using Drosophila pseudoobscura and its relatives, and many other researchers have also examined this problem by the same method using many Drosophila species (See Grossfield 1978). Regarding the latter question, Narise reported on the emigration behavior of Drosophila melanogaster. In these experiments (Narise 1966, 1968, 1969, 1974, 1978) it was shown that the emigration behavior of one strain was affected by another strain when the two were genetically different. The emigration activity of one strain is stimulated, while that of the other is diminished. Narise (1969) also reported that the emigration activity of each strain was more affected by the other when there is much genetic difference between the two mixed strains. In addition to these observations, the effect of one population on the emigration behavior of 


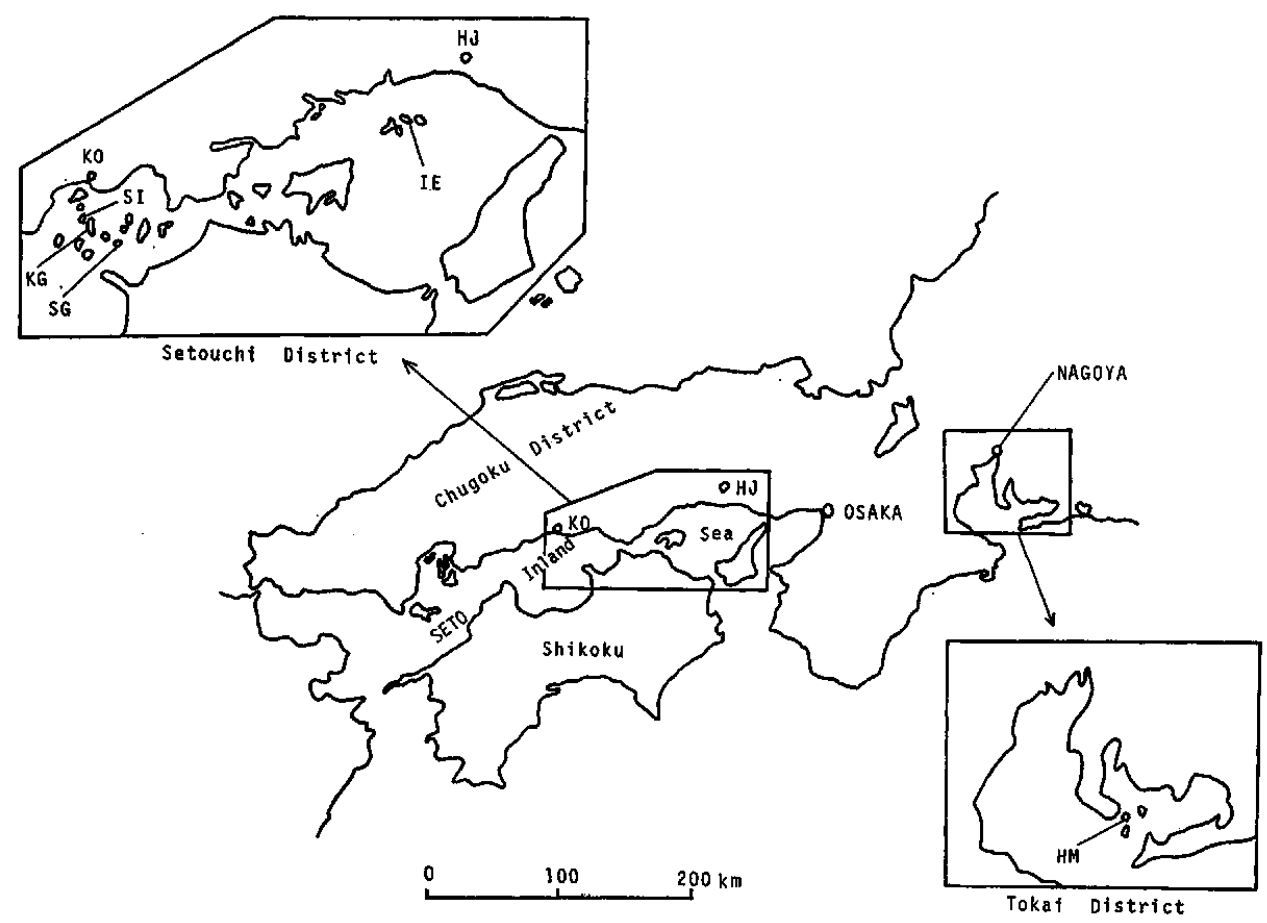

Fig. 1. The locality and abbreviation of the population in which flies were collected.

another population is also an important phenomenon in emigration.

In this experiment, the degree to which one population affects the emigration behavior of another population was studied using various strains of Drosophila melanogaster.

\section{MATERIALS AND METHODS}

Seven wild strains of Drosophila melanogaster collected from natural populations were used. The locality and abbreviation of these populations are presented in Fig. 1 and Table 1. Among them, four strains (SI, KG, SG and IE) were captured on island in the Setouchi District; two (KO and HJ) on the mainland in the Chugoku District; and (HM) on an island in the Tokai District. As seen in Fig. 1, SI, SG, KG and KO population are near with one another, and ferry boats operate about ten times per day among these islands and Kasaoka. Therefore, passive transport of flies is expected among these populations. Collected strains of flies were mass cultured separately in half-pint milk bottles for one and a half years before starting the experiments.

A total of fifty pairs of flies were introduced into a migration-tube (original tube) and kept there for 24 hours. Then, three new tubes with food were 
Table 1. Emigration activity of single strains and mixtures (Mean and standard deviation)

\begin{tabular}{|c|c|c|}
\hline \multicolumn{3}{|l|}{ Single strain } \\
\hline \multicolumn{2}{|c|}{ Shiraishijima (SI) } & $25.0 \pm 4.90$ \\
\hline \multicolumn{2}{|c|}{ Kitagishima (KG) } & $24.3 \pm 5.76$ \\
\hline \multicolumn{2}{|c|}{ Sanagishima (SG) } & $23.5 \pm 4.65$ \\
\hline \multicolumn{2}{|c|}{ Kasaoka (KO) } & $27.3 \pm 2.79$ \\
\hline \multicolumn{2}{|c|}{ Himeji (HJ) } & $26.1 \pm 2.02$ \\
\hline \multicolumn{2}{|c|}{ Ieshima (IE) } & $25.5 \pm 7.74$ \\
\hline \multicolumn{2}{|c|}{ Himagashima (HM) } & $15.5 \pm 3.60$ \\
\hline Mixture & Rel. freq. of SI & \\
\hline \multirow[t]{3}{*}{$\mathrm{SI}+\mathrm{KG}$} & 0.1 & $20.8 \pm 6.91$ \\
\hline & 0.5 & $22.8 \pm 3.11$ \\
\hline & 0.9 & $19.2 \pm 2.59$ \\
\hline \multirow[t]{2}{*}{$\mathrm{SI}+\mathrm{SG}$} & 0.1 & $23.4 \pm 1.52$ \\
\hline & 0.5 & $21.4 \pm 4.83$ \\
\hline \multirow{4}{*}{$\mathrm{SI}+\mathrm{KO}$} & 0.9 & $20.2 \pm 5.59$ \\
\hline & 0.1 & $30.6 \pm 4.67$ \\
\hline & 0.5 & $27.6 \pm 5.81$ \\
\hline & 0.9 & $22.0 \pm 7.52$ \\
\hline \multirow{3}{*}{$\mathrm{SI}+\mathrm{IE}$} & 0.1 & $21.6 \pm 3.85$ \\
\hline & 0.5 & $25.8 \pm 2.77$ \\
\hline & 0.9 & $33.2 \pm 4.76$ \\
\hline \multirow[t]{3}{*}{$\mathrm{SI}+\mathrm{HJ}$} & 0.1 & $24.0 \pm 2.35$ \\
\hline & 0.5 & $26.0 \pm 3.81$ \\
\hline & 0.9 & $26.0 \pm 2.61$ \\
\hline \multirow[t]{3}{*}{$\mathrm{SI}+\mathrm{HM}$} & 0.1 & $25.0 \pm 3.00$ \\
\hline & 0.5 & $28.6 \pm 2.79$ \\
\hline & 0.9 & $29.2 \pm 3.11$ \\
\hline Mixture & Rel. freq. of $\mathrm{KG}$ & \\
\hline \multirow[t]{3}{*}{$\mathrm{KG}+\mathrm{SG}$} & 0.1 & $23.4+1.52$ \\
\hline & 0.5 & $23.2+1.73$ \\
\hline & 0.9 & $23.0+3.16$ \\
\hline \multirow[t]{3}{*}{$\mathrm{KG}+\mathrm{KO}$} & 0.1 & $26.8+10.63$ \\
\hline & 0.5 & $26.4+3.05$ \\
\hline & 0.9 & $24.8+4.02$ \\
\hline \multirow[t]{3}{*}{$\mathrm{KG}+\mathrm{HJ}$} & 0.1 & $26.2+2.28$ \\
\hline & 0.5 & $26.6+2.07$ \\
\hline & 0.9 & $26.6+3.20$ \\
\hline \multirow[t]{3}{*}{$\mathrm{KG}+\mathrm{HM}$} & 0.1 & $25.8+1.48$ \\
\hline & 0.5 & $27.4+2.07$ \\
\hline & 0.9 & $28.4+2.33$ \\
\hline
\end{tabular}


connected to the original tube, and emigration was allowed to take place for six hours. The flies which had emigrated to the three connected tubes were counted, and the emigration activity of each strain was estimated on the basis of number of flies of that strain emigrated as a percent of those of that strain initially introduced into the original tube.

Two kinds of experiments, single and mixed, were conducted; three kinds of relative frequencies $-0.9,0.5$, and $0.1-$ of the two strains were in the mixed experiments. In order to differentiate two wild strains from each other in the mixed condition, Invisible Fluorescent Pigment No. 2210 (green color) used for black light (Japan Lamp Industrial Co. Ltd.) was employed, and no effect of the pigment on emigration behavior was observed in the experiment. To examine the effect of one population on the emigration behavior of another population, the SI and KG strain were chosen as tester strains. The experiments were conducted in a dark room at $25^{\circ} \pm 1^{\circ} \mathrm{C}$, and five replications were made for each combination and at each relative frequency.

\section{RESULTS}

The mean emigration activity and standard deviation of strains under mixed and single conditions are presented in Table 1. The emigration activities of single strains were quite different statistically from one another $\left(F_{6,23}=6.44\right.$ significant at $1 \%$ level $)$, and the activities of island populations were lower than those of inland populations. The emigration activities of mixtures between SI and neighbouring populations such as KG, SG or KO decreased with an increase in the relative frequency of SI, whereas the acti-

Table 2. Analysis of variance on emigration activity under mixed conditions

S I

\begin{tabular}{|c|c|c|}
\hline Source of variation & d.f. & m.s. \\
\hline Between combinations & 5 & $137.0333^{* *}$ \\
\hline Between frequencies & 2 & 17.7334 \\
\hline Interaction & 10 & $60.5467^{* *}$ \\
\hline Error & 72 & 17.0278 \\
\hline \multicolumn{3}{|c|}{$\mathrm{K} \mathrm{G}$} \\
\hline Source of variation & d. $f$. & m.s. \\
\hline Between combinations & $\mathbf{3}$ & $49.8834^{* *}$ \\
\hline Between frequencies & 2 & 0.6167 \\
\hline Interaction & 6 & $31.8306 * *$ \\
\hline Error & 48 & 9.5899 \\
\hline
\end{tabular}

** Significant at one per cent level 
Table 3. Emigration activity of each strain at three relative frequencies under mixed conditions (Mean and standard deviation)

\begin{tabular}{|c|c|c|c|c|c|c|}
\hline \multirow{2}{*}{ Combination } & \multicolumn{3}{|c|}{$\begin{array}{l}\text { Emigration activity of SI strain at } \\
\text { frequency of SI strain }\end{array}$} & \multicolumn{3}{|c|}{$\begin{array}{l}\text { Emigration activity of opponent strain } \\
\text { at frequency of opponent strain }\end{array}$} \\
\hline & 0.9 & 0.5 & 0.1 & 0.1 & 0.5 & 0.9 \\
\hline & $.2 \pm 0.98$ & $24.8 \pm 4.60$ & $32.0 \pm 28.60$ & $26.0 \pm 18.20$ & $20.8 \pm 5.93$ & $19.6 \pm$ \\
\hline $\mathrm{SI}+\mathrm{SG}$ & $.8 \pm 6.77$ & $19.2 \pm 4.38$ & $30.0 \pm 12.30$ & $28.0 \pm 8.37$ & $23.6 \pm 5.73$ & $20.4 \pm 2.53$ \\
\hline $\mathrm{SI}+\mathrm{KO}$ & $2 \pm 8.38$ & $19.2 \pm 4.82$ & $24.0 \pm 5$ & $38.0 \pm 4.47$ & $36.0 \pm 9.70$ & $31.8=$ \\
\hline $\mathrm{SI}+\mathrm{HJ}$ & $1 \pm 3.82$ & $26.8 \pm 6.26$ & $32.0 \pm 14$ & $42.0 \pm 13.00$ & $25.2 \pm 4.15$ & 23.0 \\
\hline $\mathrm{SI}+\mathrm{IE}$ & $.1 \pm 2.91$ & $27.6 \pm 5.37$ & $34.0 \pm 8.94$ & $68.0 \pm 13.00$ & $24.0 \pm 7.21$ & $20.2 \pm 3.64$ \\
\hline $\mathrm{SI}+\mathrm{HM}$ & $28.7 \pm 4.10$ & $42.4 \pm 7.13$ & $40.0 \pm 7.07$ & $34.0 \pm 20.70$ & $14.8 \pm 10.80$ & $23.3 \pm 2.94$ \\
\hline \multirow[t]{2}{*}{ Combination } & \multicolumn{3}{|c|}{$\begin{array}{l}\text { Emigration activity of } \mathrm{KG} \text { strain at } \\
\text { frequency of } \mathrm{KG} \text { strain }\end{array}$} & \multicolumn{3}{|c|}{$\begin{array}{l}\text { Emigration activity of opponent strain } \\
\text { at frequency of opponent strain }\end{array}$} \\
\hline & 0.9 & 0.5 & 0.1 & 0.1 & 0.5 & 0.5 \\
\hline $\mathrm{KG}+\mathrm{SG}$ & $22.5 \pm 3.96$ & $23.6 \pm 5.27$ & $28.0 \pm 4.47$ & $28.0 \pm 8.37$ & $22.8 \pm 1.60$ & $22.9 \pm 1.86$ \\
\hline $\mathrm{KG}+\mathrm{KO}$ & $23.5 \pm 3.47$ & $24.0 \pm 4.69$ & $28.0 \pm 8.95$ & $34.0 \pm 8.37$ & $28.8 \pm 4.60$ & $27.7 \pm 8.68$ \\
\hline $\mathrm{KG}+\mathrm{HJ}$ & $25.8 \pm 2.98$ & $27.0 \pm 6.62$ & $30.0 \pm 8.00$ & $34.0 \pm 9.40$ & $26.8 \pm 1.79$ & $26.9 \pm 2.14$ \\
\hline $\mathrm{KG}+\mathrm{HM}$ & $27.6 \pm 2.59$ & $27.2 \pm 2.28$ & $32.0 \pm 9.01$ & $36.0 \pm 9.52$ & $28.0 \pm 2.00$ & $25.3 \pm 2.88$ \\
\hline
\end{tabular}

vities increased with an increase in the frequency of SI when distant populations were mixed. The same tendency was detected in the mixtures of $\mathrm{KG}$ and other four strains except $\mathrm{KG}$ and $\mathrm{HJ}$ mixture. The analysis of variance on emigration activities of mixtures was made, and the results are presented in Table 2 and arcsine transformation was made for statistical analysis. Highly significant deviations were detected between combinations and between frequencies, suggesting that the activities of mixtures are different for different combinations as well as for relative frequencies in the mixtures.

Table 3 presents the emigration activity of each strain at three relative frequencies under mixed conditions. As seen in this table, the emigration activity of each strain generally increased with an increase in the relative frequency of other strain, and the activity at 0.1 is higher than that in the single condition. Regarding the SI strain, its activity at 0.9 and 0.5 diminished in comparison with activity in the single condition when mixed with neighbouring population (e.g., KG, SG, and KO), while it increased when mixed with distant populations (e.g., HJ, IE, and HM). The same was hold in the mixture of KG and other four strains. In the opponent strains of SI or $\mathrm{KG}$, the activity of each strain tended to diminish at 0.9 and 0.5 when mixed with the SI strain or KG strain. The analysis of variance on emigration activity of tester and opponent strains under mixed conditions was made separately, and the results are shown in Table 4 . Highly significant deviations were 
Table 4. Analyses of variance on emigration activity under mixed conditions

\begin{tabular}{|c|c|c|c|c|c|}
\hline \multirow{2}{*}{ Source of variation } & \multicolumn{2}{|c|}{ SI strain } & \multirow{2}{*}{ Source of variation } & \multicolumn{2}{|c|}{ Opponent strain } \\
\hline & d. f. & m.s. & & d. $f$. & m.s. \\
\hline Between combinations & $\mathbf{5}$ & $485.3689^{* *}$ & Between strains & 5 & $623.2975^{* *}$ \\
\hline Between frequencies & 2 & $591.1485^{* *}$ & Between frequencies & 2 & $2493.3778^{* *}$ \\
\hline Interaction & 10 & 53.9645 & Interaction & 10 & $542.8308^{* *}$ \\
\hline Error & 72 & 91.7934 & Error & 72 & 78.0985 \\
\hline \multirow{2}{*}{ Source of variation } & \multicolumn{2}{|c|}{$\mathrm{KG}$ strain } & \multirow{2}{*}{ Source of variation } & \multicolumn{2}{|c|}{ Opponent strain } \\
\hline & d. f. & m.s. & & d. f. & m. s. \\
\hline Between combinations & 3 & $67.4786^{* *}$ & Between strains & 3 & $103.1691^{* *}$ \\
\hline Between frequencies & 2 & $127.3482^{* *}$ & Between frequencies & 2 & $316.3180^{* *}$ \\
\hline Interaction & 6 & 8.6901 & Interaction & 6 & 7.8100 \\
\hline Error & 48 & 14.1435 & Error & 48 & 8.6242 \\
\hline
\end{tabular}

** Significant at one per cent level

Table 5. A/B ratio of each strain under mixed conditions at three relative frequencies

\begin{tabular}{|c|c|c|c|c|c|c|}
\hline \multirow{2}{*}{ Combination } & \multicolumn{3}{|c|}{ SI strain } & \multicolumn{3}{|c|}{ Opponent strain } \\
\hline & 0.9 & 0.5 & 0.1 & 0.9 & 0.5 & 0.1 \\
\hline $\mathrm{SI}+\mathrm{KG}$ & 0.73 & 0.99 & 1.28 & 0.84 & 0.86 & 1.07 \\
\hline $\mathrm{SI}+\mathrm{SG}$ & 0.79 & 0.77 & 1.20 & 0.87 & 1.00 & 1.19 \\
\hline $\mathrm{SI}+\mathrm{KO}$ & 0.81 & 0.77 & 0.96 & 1.16 & 1.31 & 1.39 \\
\hline $\mathrm{SI}+\mathrm{HJ}$ & 1.00 & 1.07 & 1.28 & 0.89 & 0.97 & 1.61 \\
\hline $\mathrm{SI}+\mathrm{IE}$ & 1.08 & 1.10 & 1.36 & 0.79 & 0.94 & 2.67 \\
\hline $\mathrm{SI}+\mathrm{HM}$ & 1.15 & 1.70 & 1.60 & 1.50 & 0.95 & 2.19 \\
\hline \multirow{2}{*}{ Combination } & \multicolumn{3}{|c|}{ KG strain } & \multicolumn{3}{|c|}{ Opponent strain } \\
\hline & 0.9 & 0.5 & 0.1 & 0.9 & 0.5 & 0.1 \\
\hline $\mathbf{K G}+\mathbf{S G}$ & 0.92 & 0.97 & 1.15 & 0.97 & 0.97 & 1.19 \\
\hline $\mathrm{KG}+\mathrm{K} 0$ & 0.96 & 0.99 & 1.15 & 1.01 & 1.05 & 1.25 \\
\hline $\mathrm{KG}+\mathrm{HJ}$ & 1.06 & 1.11 & 1.23 & 1.03 & 1.03 & 1.30 \\
\hline $\mathrm{KG}+\mathrm{HM}$ & 1.13 & 1.13 & 1.32 & 1.63 & 1.80 & 2.32 \\
\hline
\end{tabular}

detected between combinations and between frequencies in SI, and between strains, between frequencies, and interaction between frequency and strain in the opposing strains. In KG and opponent strains, highly significant deviations were detected between combinations and between frequencies. The facts indicate that the emigration activity of each strain differs under mixed 
conditions.

The A/B ratio (see Narise 1969) of each strain under the mixed condition was calculated, and the results are presented in Table 5. Here, A stands for the emigration activity of a strain under the mixed condition; and B for that in the single condition. In general, the $\mathrm{A} / \mathrm{B}$ ratio of each strain is higher than unity at 0.1 . This indicates that the strain was stimulated to emigrate by the opponent strain. It is also clear that the $\mathrm{A} / \mathrm{B}$ ratio of a strain depends on the relative frequency of the strain in the mixture. Regarding the tester strain, there is the tendency for the ratio of the tester strain to be lower than unity when the strain is mixed with neighbouring populations, while the value is more than unity when mixed with distant populations. Also, it may be seen that the more distant the two populations, the higher the $A / B$ ratio.

\section{DISCUSSION}

One of the important problems in emigration is to what extent one population affects the emigration behavior of another population.

As seen in Table 1, emigration activities of mixtures of neighbouring populations (SI, KG, SG and KO) are lower than those for the same strain in the single condition, regardless of the relative frequency of the SI strain except for the KO strain in which the activity decreased with an increase in the relative frequency of the SI strain. At 0.9 and 0.5 SI frequencies, the decrease of emigration activity in mixtures is mainly due to the diminution of the activity of the SI strain, whereas the decrease at 0.1 is due to diminution of the opponent strain activity. This decrease at 0.1 SI occurs even though the SI activity at this frequency increases (see Table 3). However, the activities of mixtures of distant populations increased with an increase in the relative frequency of the SI strain and were higher than those of the strains in the single condition when the SI strain was dominate in the mixtur. As shown in Table 3 , the stimulation of activities in these mixtures is due to interaction between the two coexisting strains. The emigration activity of the SI strains was accelerated in general by flies from distant populations, while the opponent strain activity was reduced. However, the rate of increase in SI activity is greater than the rate of decrease in opponent strain activity so that the net activity of the mixtues is increased and is related to the relative frequency of SI. In the case of the SI/HM mixture, the activities of both strains are stimulated by each other. Regarding KG and the opponent strains, the activities of mixtures of $\mathrm{KG}$ and neighbouring populations decreased with the increase of relative frequency of $\mathrm{KG}$, while increased in the mixture of KG and HM strain. The decrease or increase of the activity is mainly due to emigration behavior of $\mathrm{KG}$ strain in a mixed condition.

With regards to the $A / B$ ratio, the ratio at 0.1 relative frequency of either 
strain is higher than unity except for the $\mathrm{SI} / \mathrm{KO}$ combination indicating more frequent movement of those flies in the majority from the original tube. From the experimental results, it can be said that it is difficult for few flies from other populations to stay in the population to which they emigrate. At 0.9 and 0.5 relative frequencies, one strain in a mixture stimulates the activity, while the opponent one reduces the activity showing that the $A / B$ ratio of the former strain is higher than unity, but the ratio of the latter is lower in general.

In addition, the A/B ratio of the SI strain at SI frequency of 0.9 and 0.5 is lower than unity when it is mixed with neighbouring populations, but higher in mixtures with distant populations. The $\mathrm{A} / \mathrm{B}$ of the SI strain in SI/HM mixture is the highest, and these in SI/HS and SI/IE mixtures are intermediate as shown in Table 5.

The HM population is very far from the SI population. Two populations, the HJ and IE, are not so far as HM, and the other three are near each other as seen in Fig. 1. Therefore, the ratio seems to be related to geographical distance. Narise (1969) reported that the A/B ratio of a strain is lower when two genetically similar strains were mixed than when they were genetically different. Consequently, the HM population, which is far from the SI population geographically, is quite different genetically from it, and four populations among which possive transport of flies is expected-SI, SG, KG and KO-are similar. And IE and HJ are not so different from SI as HM. The same tendency was also observed in the mixtures of KG and other four strains. From the experimental results we propose that emigratory behavior of a population is not much affected by emigrants from neighbouring populations and we can thus use the $A / B$ ratio as a tool for measuring the genetic similarity among populations.

We thank Dr. L. D. Frye, Research Associate of Josai Dental University, for critical reading of the manuscript. This study was supported by Josai Dental University Grant No. 9 in 1981 and No. 74 in 1982.

\section{REFERENCES}

DOBZHANSKY, Th. and WRIGHT, S. (1943) Genetics of natural populations. X. Dispersion rates in Drosophila pseudoobscura. Genetics 28, 304-340.

DoBZHANSKY, Th. and WRIGHT, S. (1947) Genetics of natural populations. XV. Rate of diffusion of a mutant gene through a population of Drosophila pseudoobscura. Genetics 32, 303-324.

Grossfield, J. (1978) Non-Sexual Behavior of Drosophila. In The Genetics and Biology of Drosophila Vol. 2b, (ed. by Ashburner, M. and Wright, T. R. F.), pp. 67-74. Academic Press, London.

NARISE, T. (1966) The mode of migration of Drosophila ananassae under competitive condition. Studies in Genetics, Univ. of Texas Publ. No. 6616, 121-131.

NARISE, T. (1968) Migration and competition in Drosophila I. Competition between wild and vestigial strains of Drosophila melanogaster in a cage and migration-tube population. Evolution 22, 301-306. 
NARISE, T. (1969) Migration and competition in Drosophila II. Effect of genetic background on migratory behavior of Drosophila melanogaster. Jpn. J. Genet. 44, 297-302.

NARISE, T. (1974) Relation between dispersive behavior and fitness. Jpn. J. Genet. 49, 131-138.

NARISE, T. (1978) Mass dispersive activity in a genetically heterogeneous condition. D. I.S. 53, 152.

Powell, J.R., Dobzhansky, Th., HooK, J. E. and Wistrand, H.E. (1976) Genetics of natural populations. XLIII. Further studies on rates of dispersal of Drosophila pseudoobscura and its relatives. Genetics 82, 493-506. 